Chapter 23

\title{
Patterns of Recreational Drug Use and Harm Reduction Strategies among Women at Music Festivals: The Case of Hungary and Poland
}

\author{
Iga Kender-Jeziorska
}

\section{Introduction}

The prevalence of use of club drugs among young adults in Europe has been stable for the last decade. For example, last year's use of cocaine and amphetamine in the general population of 15-34 years olds decreased by $0.1 \%$ each, while 3,4-methylenedioxymethamphetamine (MDMA) use has increased by $0.1 \%$ (European Monitoring Centre for Drugs and Drug Addiction (EMCDDA), 2009, 2019). It is argued that recreational use of psychoactive substances has been undergoing a process of normalisation, gaining increasing societal acceptance (Parker, Williams, \& Aldridge, 2002). Although certain aspects of this theory have been criticised (e.g. Gourley, 2004; Williams, 2016), the evidence seems to support the normalisation thesis in two major contexts: among youth (Cheung \& Cheung, 2006; van der Poel, Rodenburg, Dijkstra, Stoele, \& van de Mheen, 2009; Wilson, Bryant, Holt, \& Treloar, 2010) and in party-club settings (Duff, 2005; Hutton, 2010; Ravn, 2012).

In support of the normalisation theory in party settings, the research shows show that the prevalence of illicit drug use among people participating in the nightlife economy is much higher than in the general population (EMCDDA, 2006). In Oslo, $25 \%$ of young adults surveyed have reported last month drug use (Nordfjærn, Bretteville-Jensen, Edland-Gryt, \& Gripenberg, 2016, p. 3); in Edinburgh, $35 \%$ of study participants have declared using ecstasy every week (Riley, James, Gregory, Dingle, \& Cadger, 2001, p. 1035); in Rome, 39\% of club-goers

\footnotetext{
The Impact of Global Drug Policy on Women: Shifting the Needle, 201-211

Copyright $(C) 2021$ by Iga Kender-Jeziorska

These works are published under the Creative Commons Attribution (CC BY 4.0) licence. Anyone may reproduce, distribute, translate and create derivative works of these works (for both commercial and non-commercial purposes), subject to full attribution to the original publication and authors. The full terms of this licence may be seen at http://creativecommons.org/licences/by/4.0/legalcode doi:10.1108/978-1-83982-882-920200028
} 
used illicit drugs in the last 12 hours (Vento et al, 2014, p. 3); and in several venues in the United States, almost half of research participants have been under the influence of an illicit substance at the moment of entering the club (Miller, Furr-Holden, Voas, \& Bright, 2005, p. 366).

The research on prevalence and patterns of recreational substance use primarily focusses on club settings in the United States (Ramo, Grov, Delucchi, Kelly, \& Parsons, 2010), Australia (Degenhardt, Dillon, Duff, \& Ross, 2006; P. Miller et al., 2015) and Western Europe (Hannemann, Kraus, \& Piontek, 2017; Moore, Dargan, Wood, \& Measham, 2013; Ravn, 2012). Studies of (open air) festivals are much more scarce, yet have similar geographical distribution (e.g. Day et al, 2018; Hoegberg et al., 2018; Lai et al., 2013; Martinus, McAlaney, McLaughlin, \& Smith, 2010; Mohr, Friscia, Yeakel, \& Logan, 2018). Moreover, such enquiries typically do not include other aspects which are crucial for the safety of nightlife attendees.

This chapter aims to fill a gap in the literature by examining drug use patterns and application of harm reduction measures by women attending music festivals in two countries of severely understudied East-Central European region: Hungary and Poland.

\section{Methods}

This study is part of a broader research, which also includes the sexual experiences of the respondents. In this chapter, only the drug use-related results are discussed. The aim is to provide an insight into the phenomena and reveal potential relationships between them. The research questions are as follows: What is the prevalence of drug use among women at music festivals? What types of drugs are the most prevalent? What is the intensity of drug use? What is the level of self-assessed knowledge about the composition of the used substance? What is the prevalence of using reagent drug testing? What is the prevalence of ensuring the presence of a trip-sitter? What, if any, are the relationships between the type of drug used, intensity of use and the application of harm reduction measures? Answering these questions, on a practical level, can help to identify the riskiest patterns of substance use, hence aiding professional organisations in the development of educational, preventive and harm reduction interventions in the examined settings. The geographical scope of the study is Poland and Hungary as countries representative of East-Central Europe.

The data was collected over one month during the summer of 2017. The questionnaire (translated into both languages) included 53 questions: 4 about demographic characteristics, 20 about substance use and harm reduction measures and 29 about sexual experiences. The questions regarding patterns of drug use were of qualitative and descriptive character and did not ask about the number of occasions or amount of substances used. Since the effects of drugs can vary not only from one person to another but also for the same person depending on the settings and their physical and psycho-emotional state at the time of consumption, subjective experience of these effects was the measure adopted. The utility of the results for harm reduction professionals also motivates this choice: 
regardless of how much of a substance a person digested in absolute terms, the (lack of) need for the intervention depends on the emotional and psycho-spiritual state of the individual. For example, the intensity of alcohol use was measured on a scale from 'none' to 'I drink as much as I can, sometimes I experience blackouts'; the intensity of cocaine use was measured on a scale from 'none, to 'I use enough to have much energy at all times and significantly altered perception. During festivals, I sleep very little'. All questions included the 'I prefer not to answer' option.

Convenience sampling was used to reach out to respondents. The invitation to participate was distributed on social media pages of major Polish and Hungarian festivals (representing various music genres) and through other social media channels. Participation was based on self-selection, voluntary, anonymous and not related to any material gratification. A total of 1006 respondents answered the invitation, and 510 complete responses were collected. The analysis was performed on the aggregate data from two countries. The Spearman correlation test was used to reveal relationships between variables.

\section{Results}

\section{Sample Characteristics}

Out of 510 respondents who completed the drug-related part of the questionnaire, 297 (58\%) were Polish and 213 (42\%) were Hungarian speakers. The respondents were aged from 14 to 56 years old, with a mean age of 25.9 years. First festival attendance ('festival initiation') occurred on average at the age of 17.9 years (min. 2 years old, max. 55 years old). The respondents attended from one to four festivals annually $(M=2.3)$, with $55 \%$ of them attending two. Over two-thirds of the research participants started to use alcohol regularly (several times a month) before reaching the legal drinking age, being 14-17 years old.

Regarding general substance use, all of the respondents had consumed alcohol and $77 \%$ of them did so during the last week. Concerning illicit drugs, Table 1 summarises the general patterns of substance use among studied women $(N=510)$.

Table 1. The Prevalence of Substance Use among Female Festivalgoers.

\begin{tabular}{lccccc}
\hline & $\begin{array}{c}\text { Never } \\
\mathbf{( \% )}\end{array}$ & $\begin{array}{c}\text { Lifetime } \\
\mathbf{( \% )}\end{array}$ & $\begin{array}{c}\text { Last Year } \\
\mathbf{( \% )}\end{array}$ & $\begin{array}{c}\text { Last Month } \\
\mathbf{( \% )}\end{array}$ & $\begin{array}{c}\text { Last Week } \\
\mathbf{( \% )}\end{array}$ \\
\hline Alcohol & 0 & 3 & 5 & 15 & 77 \\
Cannabis & 7 & 15 & 12 & 22 & 44 \\
Amphetamine & 40 & 18 & 13 & 16 & 13 \\
Cocaine & 58 & 17 & 11 & 8 & 6 \\
MDMA & 35 & 13 & 15 & 20 & 17 \\
LSD & 53 & 12 & 10 & 14 & 11 \\
Mushrooms & 55 & 18 & 14 & 9 & 4 \\
\hline
\end{tabular}


As many as $93 \%$ of the respondents reported ever using cannabis, with almost half of them. The majority of the respondents also had used amphetamine and MDMA in their lifetime, while nearly half had tried lysergic acid diethylamide (LSD) and psilocybin mushrooms. The overall prevalence of cocaine use was the lowest. Around $37 \%$ of the respondents recently (within the past month or week) had used MDMA, while $29 \%$ amphetamine. The lowest prevalence of recent use was related to psilocybin, where only one in eight respondents reported use.

\section{Patterns of Drug Use in Festival Settings}

The vast majority $(96 \%)$ of women taking part in the research do use mind-altering substances during music festivals they attend. As shown in Fig. 1, the most popular drug consumed during festivals is alcohol, consumed by nearly nine in ten women.

Cannabis, used by two-thirds of respondents, was the second most prevalent substance, followed by MDMA, used by every second person. Nearly one-third of women used LSD at festivals and one in five used psilocybin. Cocaine use prevalence is the lowest. Beside substances listed in the survey, a limited number of women declare using other drugs, primarily New Psychoactive Substance (NPS) (stimulants or MDMA derivatives), ketamine and DMT (N, N-dimethyltryptamine).

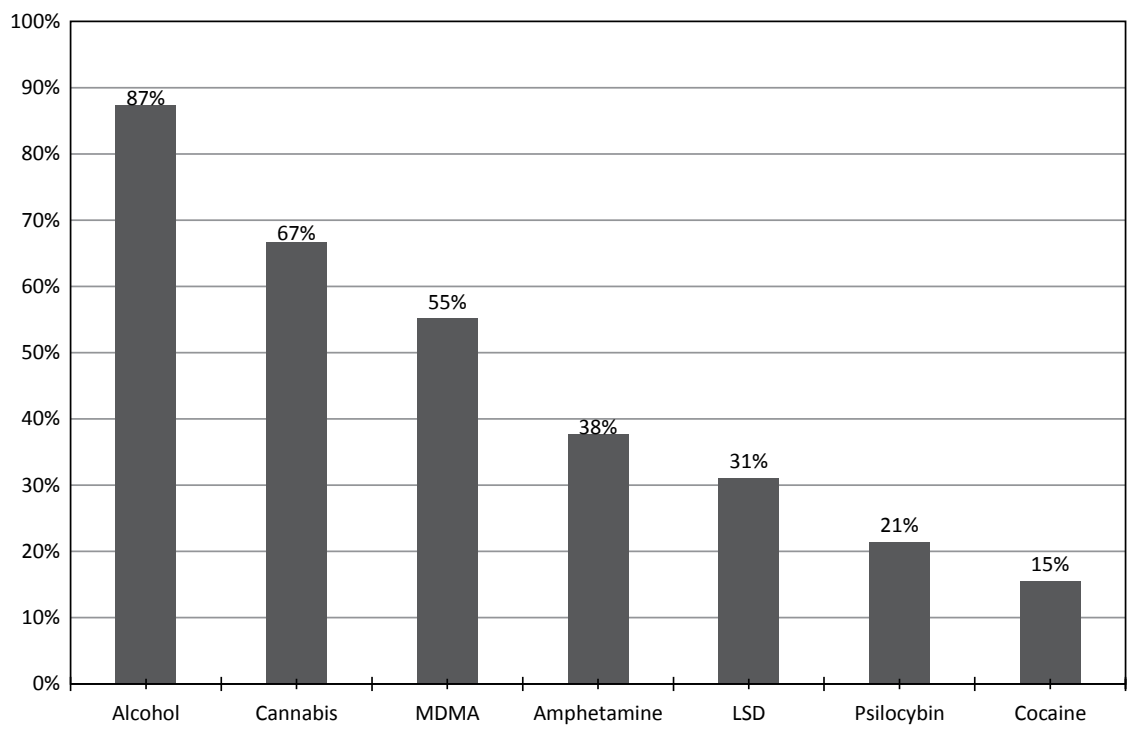

Fig. 1. The Substances Consumed by Women during a Festival $(N=510)$

Source: Author's online survey data. 
On an aggregate level for all examined drugs, moderate use seems to be dominant, with over $40 \%$ of women reporting it. Light use is characteristic for one-fourth of women, while moderately heavy use for one-fifth. Only one in ten women report heavy drug use at festivals. Around $73 \%$ of respondents admit to mixing substances, using two or more drugs simultaneously, with almost half of them doing so often or very often.

Alcohol Slightly over one in ten women $(\mathrm{N}=510)$ declared no consumption of alcohol while at a festival. At the other extreme, $4 \%$ of those drinking $(N=445)$ declared heavy drinking, with possible blackouts. Around $52 \%$ of the respondents reported moderate drinking ('feeling tipsy'), while one in five moderately heavy ('feeling drunk'). Light drinking ('no visible effect') is also relatively prevalent $(24 \%)$. Among those women who drink alcohol at festivals $(N=445)$, the majority also use cannabis $(69 \%)$ and MDMA (55\%). A large proportion of them also uses amphetamine $(38 \%)$ and LSD $(30 \%)$. The lowest festival-use prevalence among drinking women is characteristic for psilocybin (21\%) and cocaine $(17 \%)$. Three-quarters declare mixing substances. Intensified alcohol consumption in this group is very weakly positively associated with intensified cannabis consumption $(\mathrm{rs}(444)=0.110, p=0.020)$ and negatively associated with intensified LSD consumption ( $\mathrm{rs}(444)=-0.107, p=0.024)$. That is, increased alcohol consumption favours increased cannabis consumption and decreased LSD consumption.

Cannabis Exactly two-thirds of women in the survey used cannabis and its derivatives during festivals. Among them $(N=340)$, the vast majority reported light ('feeling slight effect') or moderate ('feeling relaxed') use ( $39 \%$ each). One in six women smoked to the point that they 'sometimes need a moment to get their head together' (moderately heavy use). Heavy use ('smoking often, not feeling like getting up and doing anything') was sporadic, with $6 \%$ of respondents reporting it in festival settings.

Among women declaring the smoking of cannabis at festivals, popular substances also used in festival settings included alcohol (90\%), MDMA (66\%), amphetamine (44\%) and LSD (39\%). The use of psilocybin (28\%) and cocaine $(18 \%)$ was less prevalent. Nine in ten women smoking cannabis reported using more than one drug at the same time and $50 \%$ of them did it often or very often.

Within this group, intensified cannabis consumption at festivals is very weakly or weakly correlated with intensified consumption of other substances: amphetamine $(\mathrm{rs}(339)=0.122, p=0.025)$, MDMA $(\mathrm{rs}(339)=0.122, p=0.025)$, alcohol $(\mathrm{rs}(339)=0.142, p<0.001), \operatorname{LSD}(\mathrm{rs}(339)=0.216, p<0.001)$ and psilocybin $(\mathrm{rs}(339)=0.210, p<0.001)$. Besides, more intense use of cannabis favours mixing substances, although the relationship is weak $(\mathrm{rs}(339)=0.238, p<0.001)$.

Amphetamine Around $38 \%$ of women reported using amphetamine while at a festival. Within this group ( $N=192)$, moderate use dominated ('not to feel fatigued for a longer time') and was reported by slightly more than half of women surveyed. One in five women reported light use ('slight effect'), however, also one in five women admitted heavy use, involving amounts that ensured a consistently 
high level of energy and significantly altered perception. At the same time, they slept little during festivals.

The majority of women using amphetamine also used alcohol $(88 \%)$, MDMA (87\%) and cannabis (78\%). Almost every second one, besides amphetamine, consumed LSD, nearly one in three cocaine $(32 \%)$ or psilocybin $(33 \%)$. Nearly all respondents $(94 \%)$ declared the use of two or more substances simultaneously.

Increased amphetamine consumption within this group was moderately related to increased MDMA consumption $(\mathrm{rs}(191)=0.424, p<0.001)$, which is to say the more amphetamine used the more likely it was for MDMA use to be higher. Very weak positive association is observed with increased cannabis $(\mathrm{rs}(191)=0.164, p=0.023)$ and cocaine $(\mathrm{rs}(191)=0.156), p=0.031)$ use. Increased amphetamine use also correlated with the mixing of substances $(\mathrm{rs}(191)=0.271$, $p<0.001)$.

Cocaine The majority of women surveyed $(83 \%)$ did not use cocaine while partying at festivals. Among those who did $(N=79)$, one in five reported heavy use, with the remainder characterised by primarily light (39\%)and moderate( $38 \%$ use. Moderately heavy use ('having difficulties controlling behaviour') was marginal, with only $3 \%$ of women who used cocaine reporting this frequency of use.

Within the group of women using cocaine, the majority used all other examined substances, except for psychedelics: LSD and psilocybin were used by $48 \%$ and $41 \%$ of respondents respectively. The most popular second substance in this group was alcohol (94\%), followed by MDMA (85\%), amphetamine $(79 \%)$ and cannabis $(76 \%)$. Almost all women using cocaine reported using two or more substances at the same time.

Increased cocaine use in this group was moderately associated with increased MDMA use $(\operatorname{rs}(78)=0.398, p<0.001)$ and weakly associated with the increased use of amphetamine $(\operatorname{rs}(78)=0.379, p=0.001)$, although the strength of the latter relationship verged on a moderate level. Higher intensity of cocaine use also favoured mixing substances $(\mathrm{rs}(78)=0.274, p=0.015)$.

MDMA MDMA was used by the majority (55\%) of women surveyed. Within this group $(N=281), 39 \%$ reported moderate ('not to sleep and to be able to dance all night in a good mood') and 38\% moderately heavy use ('intense euphoria and unity with others, significantly altered perception, possibly blurred sight, coordination of movements challenging'). Light use ('slight improvement of mood and increase in energy level') was a characteristic of $17 \%$ of women using MDMA at festivals. In turn, heavy use was reported by only one in 20 women ('sometimes feeling bad, wishing the drug stopped working, possible problems with walking and sharp sight, strong jaw clench, poor control of behaviour').

The majority of women using MDMA also used alcohol (87\%) and cannabis $(79 \%)$. By contrast, cocaine was the least prevalent, with a quarter of MDMAusing women consuming it while at a festival. Amphetamine was used by almost $60 \%$ of respondents, while LSD and psilocybin were used by $49 \%$ and $34 \%$, respectively. Nine out of ten women declared mixing substances. 
Increased MDMA consumption in this group is associated with the increased use of only one of the examined substances: amphetamine $(\mathrm{rs}(280)=0.263$, $p<0.001$ ), and the relationship is weak. A very weak positive relationship is also observed between intensified use of MDMA and using more drugs simultaneously $(\mathrm{rs}(280)=0.171, p=0.004)$.

LSD Almost one-third of women reported LSD use when they attend music festivals. The intensity of its use in this group $(N=158)$ was primarily moderately heavy (44\%) ('strong visual effects, significant alteration of perception, enabling looking into oneself once in a while'). Moderate use ('noticeable change of perception and mild visual effects') was a characteristic for one in three women. Light use ('on the border of an active dose, only slight mood improvement') was reported by $11 \%$ as was heavy use ('very strong visual effects, dramatic change of perception, focus on oneself one's inside and journey, barely any connection to physical environment').

The most prevalent other drugs for women using LSD were MDMA (87\%) and cannabis (84\%). Slightly over half of the women respondents used amphetamine $(55 \%)$ and psilocybin $(52 \%)$. Only $24 \%$ reported cocaine use. More than nine out of ten women used LSD mixed substances (94\%).

Increased LSD use in this group is weakly related to increased use of cannabis $(\operatorname{rs}(157)=0.351, p<0.011)$, psilocybin $(\mathrm{rs}(157)=0.234, p=0.003)$ and cocaine $(\mathrm{rs}(157)=0.202, p=0.011)$. Higher intensity of the consumption of LSD at festivals also favours mixing drugs $(\mathrm{rs}(157)=0.217, p=0.006)$.

Psilocybin Only one in five women used psilocybin at music festivals. Within this group $(N=109), 44 \%$ declared moderate use ('noticeable change of perception and mild visual effects'). One in four women reported light use ('amount on the border of an active dose'), and the same proportion declared moderately heavy use ('strong visual effects, significant alteration of perception, enabling looking into oneself once in a while'). Heavy use was a characteristic for less than onetenth of respondents $(7 \%)$.

MDMA was the most prevalently used substance among women using psilocybin at festivals (88\%). Other substances included cannabis (86\%), alcohol (84\%) and LSD (75\%). Stimulants were not so popular, with amphetamine used by $58 \%$ and cocaine by $29 \%$ of respondents. Nearly $95 \%$ of women in this group reported using two or more drugs at the same time.

Moderate correlation is observed between intensified psilocybin use and intensified LSD use $(\mathrm{rs}(108)=0.448, p<0.001)$. There was a weak correlation with increased consumption of alcohol $(\mathrm{rs}(108)=0.360, p<0.001)$, cannabis $(\mathrm{rs}(208)=0.323, p=0.001)$, amphetamine $(\mathrm{rs}(108)=0.287, p=0.003)$ and cocaine $(\mathrm{rs}(108)=0.281, p=0.003)$, as well as mixing of drugs $(\mathrm{rs}(108)=0.367, p<0.001)$.

\section{Substance Knowledge and Harm Reduction Measures}

Half of the women who shared information $(N=423)$ reported that they always know what illegal substances they are consuming at festivals. Over a third declared that they often knew the composition of the substance. 
Among women who used illicit drugs other than cannabis $(N=327), 66 \%$ never tested their substances. Still, nearly half declared knowledge of the drugs consumed. In this group, one woman out of ten tested their substances always or often, and $22 \%$ rarely or sometimes. The relationship between the frequency of testing drugs and subjective assessment of actual substance composition was positive but very weak $(\mathrm{rs}(326)=0.113, p=0.041)$.

Around $36 \%$ of the respondents $(N=423)$ never ensured the presence of a tripsitter, the same proportion only rarely or sometimes, and $29 \%$ often or always ensured a sober person was available. In the group of women using illicit drugs other than cannabis, the numbers were similar: $34 \%$ of them never ensured trip sitting, $37 \%$ rarely or sometimes and $28 \%$ always ( $1 \%$ 'prefer not to say' answers).

Of the 492 women declaring substance use at festivals, late alcohol initiation $(\operatorname{rs}(491)=0.158, p<0.001)$, late illicit drug initiation $(\mathrm{rs}(491)=0.282, p<0.001)$ and late festival initiation $(\mathrm{rs}(491)=0.142, p=0.002)$ favoured testing their drugs. A similar correlation was observed for ensuring the presence of a trip-sitter: very weak association was observed for late alcohol initiation $(\operatorname{rs}(491)=0.170$, $p<0.001)$, late illicit drug initiation $(\mathrm{rs}(491)=0.296, p<0.001)$ and late festival initiation $(\mathrm{rs}(491)=0.100, p=0.026)$. In general, older women tend to test their substances $(\mathrm{rs}(491)=0.174, p<0.001)$ and ensure trip sitting $(\operatorname{rs}(491)=0.108, p=0.016)$ more often.

However, in many cases, more intense use of drugs at festivals was negatively correlated with adopting harm reduction measures. Testing substances was less frequent among women consuming more amphetamine $(\mathrm{rs}(491)=-0.122$, $p=0.007)$, alcohol $(\mathrm{rs}(491)=-0.133, p=0.003), \operatorname{MDMA}(\mathrm{rs}(491)=-0.177)$ and cannabis $(\operatorname{rs}(491)=-0.376, p<0.001)$ (weak relationship for cannabis and very weak for the other drugs). With respect to taking care of trip sitting, a very weak negative relationships was present in case of psilocybin $(\operatorname{rs}(491)=-0.102, p=0.023)$, cocaine $(\operatorname{rs}(491)=-0.145, p=0.001)$, amphetamine $(\operatorname{rs}(491)=-0.175, p<0.001)$ and LSD $(\mathrm{rs}(491)=-0.182, p<0.001)$. Further, weak relationships can be observed with increased consumption of $\operatorname{MDMA}(\operatorname{rs}(491)=-0.274, p<0.001)$ and cannabis $(\operatorname{rs}(491)=-0.317, p<0.001)$. The more of the above mentioned substance a woman consumed during a festival, the less likely she was to ensure the presence of a sober companion.

A moderately strong relationship is observed between the frequency of adopting the two harm reduction measures $(\operatorname{rs}(491)=0.540, p<0.001)$, that is, women who test their drugs more often, tend to take care of a trip-sitter's presence more often as well.

\section{Patterns of Drug Use in a Comparative Harm Reduction Perspective}

Table 2 presents a summary of prevalence and intensity of use for each examined substance.

Looking at each drug separately, it seems that women use psychoactive substances at festivals in a moderate way. What is concerning from a harm reduction 
Table 2. Patterns of Drug Use among Women at Festivals.

\begin{tabular}{lccrrrrr}
\hline & $\begin{array}{c}\text { Alcohol Cannabis } \\
(\mathbf{\%})\end{array}$ & $\mathbf{( \% )}$ & $\mathbf{( \% )}$ & $\mathbf{( \% )}$ & $\mathbf{( \% )}$ & $\mathbf{( \% )}$ & $\mathbf{( \% )}$ \\
\hline None & 12 & 33 & 61 & 83 & 44 & 68 & 77 \\
Light & 21 & 26 & 8 & 6 & 10 & 3 & 5 \\
Moderate & 45 & 26 & 20 & 6 & 21 & 11 & 9 \\
Moderately & 18 & 11 & 2 & 0 & 21 & 14 & 5 \\
heavy & & & & & & & \\
Heavy & 4 & 4 & 8 & 3 & 3 & 3 & 2 \\
\hline
\end{tabular}

perspective is the prevalence of heavy amphetamine use. The results suggest that almost one in ten women attending a festival consumed it in amounts resulting in intensive effect with related risks associated with sleep deprivation and/or other adverse effects of prolonged stimulant use. Besides, depending on set and setting (a person's psycho-emotional well-being during consumption of drugs and the person's environment), people using MDMA moderately heavily and - to a lesser extent - moderately (also groups of considerable sizes) may need the assistance of harm reduction professionals.

Besides the intensity of use of each substance, the variety of drugs consumed is significant (Fig. 2).

The most substantial proportion of women use two drugs (including alcohol) at a festival. However, almost one in five women used four different substances, which, depending on what they are and how they are used, can qualify as high-risk use. Moreover, every tenth woman used six or seven drugs during an event - a number that may in certain circumstances result in serious and negative consequences.

Generally, it is not advised to mix stimulants with alcohol (stimulants reducing the effects of alcohol resulting in more intense drinking and increased dehydration), with other varieties of stimulant drugs (impacts on heart rate and blood pressure) or combining stimulants, which may induce anxiety, with psychedelics as this can result in unpleasant psycho-emotional experiences. Combining psychedelics with cannabis can result in negative experiences due to unpredictable synergy between substances. Especially dangerous is the combination of cocaine and alcohol, which is metabolised to cocaethylene, which has a more negative effect on the heart than that of cocaine alone (Tripsit, 2019).

Table 3 presents the proportion of women declaring the consumption of combinations of listed substances and using various substances at the same time with varying frequency $(N=510)$. This does not mean that the numbers below show the proportion of respondents factually mixing listed drugs. They are a proxy measure shedding some light on the possibility of listed combinations and their prevalence. 


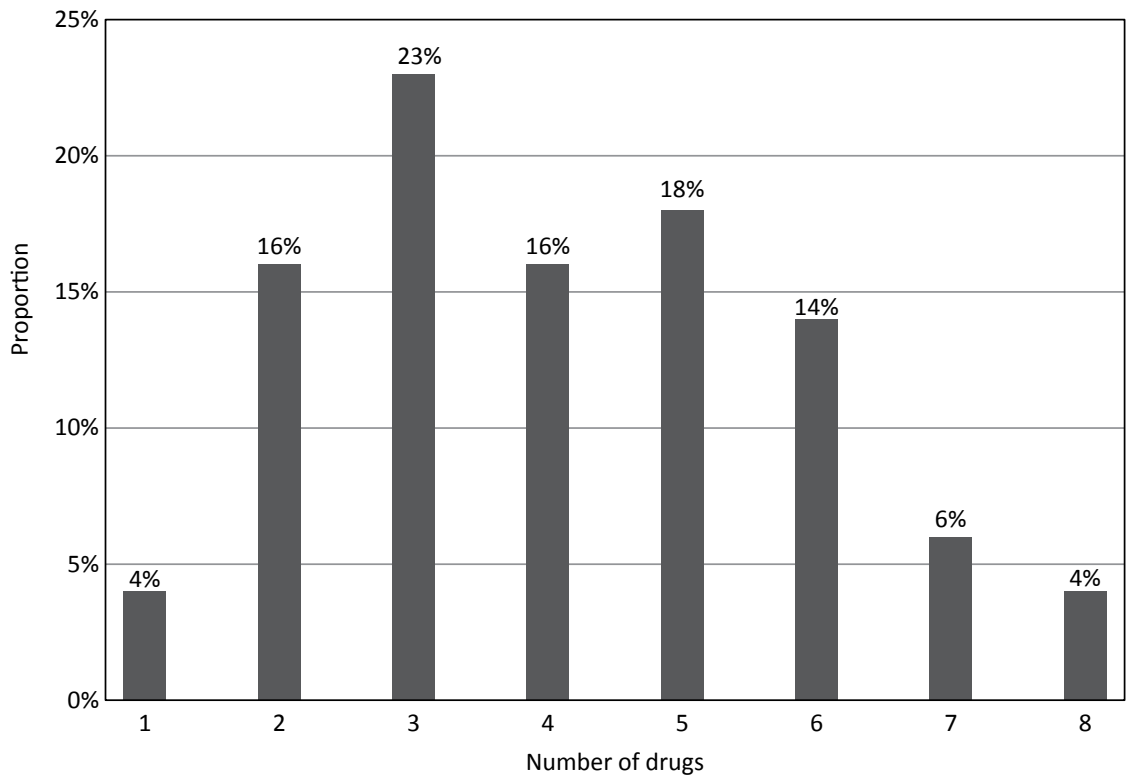

Fig. 2. The Number of Different Substances Used by Women at a Festival $(N=510)$

Table 3. Substances Used during a Festival by Women Who Tend to Mix Drugs.

Drugs Used During a Festival

Alcohol and amphetamine

Alcohol and cocaine

Amphetamine and cocaine

Amphetamine and LSD

Amphetamine and psilocybin

Cocaine and LSD

Cocaine and psilocybin

Cannabis and LSD

Cannabis and psilocybin
Proportion of Women (\%)
14

12

17

15

7

6

25

18

Final considerations relate to the adoption of harm reduction measures by female festival attendees. Among women using amphetamine and/or cocaine and/ or MDMA and/or LSD and/or psilocybin at festivals $(N=327)$, ensuring that the presence of a trip-sitter is significantly more prevalent than drug testing. While the former is practised by the majority of women (roughly two-thirds), substance testing is exercised by only one in three, which may suggest the need for more intense efforts in this area of drug education and harm reduction. 


\section{Conclusion}

The research sheds light on the severely understudied area of drug use among Hungarian and Polish (speaking) women at music festivals in East and Central Europe. The data show that the festival prevalence of substance use in the studied population is exceptionally high, with over $80 \%$ of women using illicit drugs at such events. The most popular illegal substances are cannabis and MDMA. Cocaine use is the least prevalent, in line with general trends in the region's drug market (amphetamine or simulant-type NPS are much more accessible and popular than cocaine). The vast majority of female festival attendees consume alcohol.

The results demonstrate that intensity of substance use at festivals is not excessive - heavy use of any of the examined substances is marginal (except for heavy use of amphetamine, which is more prevalent). The majority of respondents using various substances do it moderately. Nevertheless, there is a significant number of women using many different substances during one event. This may suggest a high probability of combining substances in this group, that is, using two or more drugs simultaneously, especially given that the prevalence of mixing is high.

The data show weak but numerous relationships between the intensity in the use of various drugs, revealing interesting patterns. Simplistically, it can be said that 'increased consumption favours increased consumption'. One can suspect the existence of a group of women who use larger amounts of various substances than others. It can also point to possible popular combinations. Importantly, increased use of each examined illicit substance is also correlated with an increase in the frequency of mixing drugs. It may imply that there is a group of women who are more experienced with substance use. However, although this research does not attempt to draw any causal relationships, it can also mean that combining substances is a result of a higher level of intoxication. If the latter is true, it can be a potentially important area of work for harm reduction professionals.

Finally, the results show some discrepancies between the self-assessed level of knowledge of used illicit drugs composition and actual examination of their content. The majority of women are certain about the composition of the substance they use, yet they do not test their drugs. It can result in a false feeling of safety and potentially increase the risk of adverse consequences of drug use. Low prevalence of testing drugs may be a result of insufficient drug education but, more likely, it is due to the rather unfavourable policy environment around drugs, especially in Hungary. The positive relationship between the frequency of implementing the examined harm reduction measures of testing and trip sitting may suggest the existence of two distinctive groups - one generally practicing harm reduction, and one practicing it in a limited way (or not at all). Given all of the above, it is advised for harm reduction organisations to accelerate their efforts in the area of drug education, implementation of harm reduction and promotion of available forms of testing drugs. Festival organisers should ensure the presence of harm reduction organisations at their events and create the safest possible conditions. Decision-makers, in turn, should provide appropriate legal and policy environment for such initiatives, informed by the best international practices and based on evidence and public health considerations. 